\title{
BARYON SPECTRUM AND CHIRAL DYNAMICS
}

\author{
L. Ya. GLOZMAN \\ Institute for Theoretical Physics, University of Graz, 8010 Graz, Austria \\ Alma-Ata Power Engineering Institute, 480013 Alma-Ata, Kazakhstan
}

\section{ABSTRACT}

New results on baryon structure and spectrum developed in collaboration with Dan Riska [1, 2, 3, 4] are reported. The main idea is that beyond the chiral symmetry spontaneous breaking scale light and strange baryons should be considered as systems of three constituent quarks with an effective confining interaction and a chiral interaction that is mediated by the octet of Goldstone bosons (pseudoscalar तmesons) between the constituent quarks.

\section{INTRODUCTION}

It is well known that at low temperature and density the approximate chiral symmetry of QCD is realized in the hidden Nambu-Goldstone mode. The hidden mode of chiral symmetry is revealed by the existence of the octet of pseudoscalar mesons of low mass, which represent the associated approximate Goldstone bosons. The $\eta^{\prime}$ (the $S U(3)$-singlet) decouples from the original nonet because of the $U(1)$ anomaly [5, 6]. Another consequence of the spontaneous breaking of the approximate chiral symmetry of QCD is that the valence quarks acquire their dynamical or constituent mass [7, 8, 9, [10] through Their interactions with the collective excitations of the QCD vacuum- the quark-antiquark excitations and the instantons.

We have recently suggested [1, 2] that beyond the chiral symmetry spontaneous breaking scale a baryon - should be considered as a system of three constituent quarks with an effective quark-quark interaction that is formed of a central confining part, assumed for simplicity to be harmonic, and a chiral interaction that is mediated by the octet of pseudoscalar mesons between the constituent quarks.

The simplest representation of the most important component of the interaction of the constituent quarks that is mediated by the octet of pseudoscalar bosons in the $S U(3)_{F}$ invariant limit is

$$
H_{\chi} \sim-\sum_{i<j} V\left(\vec{r}_{i j}\right) \vec{\lambda}_{i}^{F} \cdot \vec{\lambda}_{j}^{F} \vec{\sigma}_{i} \cdot \vec{\sigma}_{j}
$$

Here the $\left\{\vec{\lambda}_{i}^{F}\right\}$ :s are flavor $S U(3)$ Gell-Mann matrices and the $i, j$ sums run over the constituent quarks.

Because of the flavor dependent factor $\vec{\lambda}_{i}^{F} \cdot \vec{\lambda}_{j}^{F}$ the chiral boson exchange interaction (1.1) will lead to orderings of the positive and negative parity states in the baryon spectra, which agree with the observed ones in all sectors. In the case of the spectrum of the nucleon and $\Delta$ the strength of the chiral interaction between the constituent quarks is sufficient to shift the lowest positive parity states in the $N=2$ band (the $N(1440)$ and $\Delta(1600)$ ) below the negative parity states in the $N=1$ band $(N(1520)-N(1535)$ and $\Delta(1620)-\Delta(1700))$. In the spectrum of the $\Lambda$ on the other hand it is the negative parity flavor singlet states (the $\Lambda(1405)-\Lambda(1520)$ ) that remain the lowest lying resonances, again in agreement with experiment. The mass splittings between the baryons with different strangeness and between the $\Lambda$ 
of the $S U(3)_{F}$ symmetry that is caused by the mass splitting of the pseudoscalar meson octet and the different masses of the $\mathrm{u}, \mathrm{d}$ and the s quarks.

There is very good analogy to solid state physics. As soon as we talk about dynamical objects constituent quarks - we should forget about original QCD degrees of freedom (one gluon exchange and instanton induced interactions) like in the solid state we describe the electric and thermo properties of metals in terms of heavy dynamical electrons (cf. constituent quarks), phonons, which are Goldstone excitations in the lattice (cf. pseudoscalar mesons), and electron-phonon interaction (cf. constituent quark - pseudoscalar meson vertex). The one gluon exchange or instanton-induced interactions between the constituent quarks would correspond to the Coulomb interaction between the heavy electrons in the lattice which is known to be totally unessential.

This talk has the following structure. Section 2 below contains the proof of why the commonly used perturbative gluon exchange interaction between the constituent quarks leads to incorrect ordering of positive and negative parity states in the spectra. In section 3 we present a short historical scetch on the role of chiral symmetry in the quark based models. Section 4 contains a description of the chiral boson exchange interaction (1.1). In section 5 we describe the spectra of the nucleon, the $\Delta$ resonance and the $\Lambda$ hyperon as they are predicted with the $S U(3)_{F}$ symmetric interaction (1.1). The effect of the $S U(3)_{F}$ breaking in the interaction is considered in section 6 . In section 7 we discuss the role of the exchange current corrections to the baryon magnetic moments that are associated with the pseudoscalar exchange interaction. Section 8 contains an explanation of the $N^{*} \rightarrow N \eta\left(\Lambda^{*} \rightarrow \Lambda \eta\right.$ and $\left.\Sigma^{*} \rightarrow \Sigma \eta\right)$ branching ratios within the chiral quark model above. Finally, in section 9 a conclusion is presented.

\section{WHY DOES THE GLUON EXCHANGE BEAR NO RELATION TO BARYON SPECTRUM}

It was accepted by many people (but not by all) that the fine splittings in the baryon spectrum are due to the gluon-exchange interaction between the constituent quarks [11, 12]. Now I shall address to formal consideration of why the one gluon exchange interaction cannot be relevant to the baryon spectrum.

The most important component of the one gluon exchange interaction [1] is so called color-magnetic interaction

$$
H_{c m} \sim-\alpha_{s} \sum_{i<j} \frac{\pi}{6 m_{i} m_{j}} \vec{\lambda}_{i}^{C} \cdot \vec{\lambda}_{j}^{C} \vec{\sigma}_{i} \cdot \vec{\sigma}_{j} \delta\left(\vec{r}_{i j}\right)
$$

where the $\left\{\vec{\lambda}_{i}^{C}\right\}:$ s are color $S U(3)$ matrices. It is the permutational color-spin symmetry of the $3 \mathrm{q}$ state which is mostly responsible for the contribution of the interaction (2.1). The corresponding two-body matrix element is

$$
<\left[f_{i j}\right]_{C} \times\left[f_{i j}\right]_{S}:\left[f_{i j}\right]_{C S}\left|\vec{\lambda}_{i}^{C} \cdot \vec{\lambda}_{j}^{C} \vec{\sigma}_{i} \cdot \vec{\sigma}_{j}\right|\left[f_{i j}\right]_{C} \times\left[f_{i j}\right]_{S}:\left[f_{i j}\right]_{C S}>=\left\{\begin{array}{rr}
8 & {[11]_{C},[11]_{S}:[2]_{C S}} \\
-\frac{8}{3} & {[11]_{C},[2]_{S}:[11]_{C S}}
\end{array} .\right.
$$

Thus the symmetrical color-spin pairs (i.e. with the $[2]_{C S}$ Young pattern) experience an attractive contribution while the antisymmetrical ones $\left([11]_{C S}\right)$ experience a repulsive contribution. Hence the color-magnetic contribution to the $\Delta$ state $\left([111]_{C S}\right)$ is more repulsive than to the nucleon $\left([21]_{C S}\right)$ and the $\Delta$ becomes heavier than the nucleon. The prise is that $\alpha_{s}$ should be larger than unity, which is bad.

This interaction does not practically contribute to the $N(1535)-N$ splitting as both these states have identical mixed color-spin symmetry. Hence this splitting within this model should be due to the spinindependent confining forces which means that $\hbar \omega \simeq 500-600 \mathrm{MeV}$. This large value of the harmonic oscillator parameter implies a very small value for the nucleon radius, $\sqrt{\left\langle r^{2}\right\rangle}=\sqrt{\hbar / m \omega} \simeq 0.5 \mathrm{fm}$, if the light quark constituent mass is taken to be 330-340 MeV, as suggested by the magnetic moments of the nucleon. 
negative parity states in the spectra of the nucleon and the $\Delta$ resonance on the one hand, and the $\Lambda$ hyperon on the other. Indeed, the positive parity state $N(1440)$ and the negative parity one $N(1535)$ have the same mixed $\left([21]_{C S}\right)$ color-spin symmetry thus the color-magnetic contribution to these states cannot be very different. But the $N(1440)$ state belongs to the $N=2$ shell while the $N(1535)$ resonance is a member of the $\mathrm{N}=1$ band which means that the $N(1440)$ should lie approximately $\hbar \omega$ above the $N(1535)$. In the $\Delta$ spectrum the situation is even more dramatic. The $\Delta(1600)$ positive parity state has completely antisymmetrical CS-Young pattern $\left([111]_{C S}\right)$, while the negative parity state $\Delta(1700)$ has the mixed one. Thus the color-magnetic contribution to the $\Delta(1600)$ is much more repulsive than to the $\Delta(1700)$. In addition the $\Delta(1600)$ is the $N=2$ state while the $\Delta(1700)$ belongs to the $N=1$ band. As a consequence the $\Delta(1600)$ must lie much higher than the $\Delta(1700)$. In the spectrum of the $\Lambda$-hyperon on the other hand it is the negative parity states $\Lambda(1405)-\Lambda(1520)$ that remain the lowest lying resonances.

Finally, there is no empirical indications in the spectrum for the large spin-orbit component of the gluon-exchange interaction [11].

\section{CHIRAL SYMMETRY AND THE QUARK MODEL (HISTORICAL SCETCH)}

The importance of the constraints posed by chiral symmetry for the quark bag [13] and bag-like [14] models for the baryons was recognized early on. In the bag or bag-like models with restored chiral symmetry the massless current quarks within the bag were assumed to interact not only by perturbative gluon exchange but also through chiral meson field exchange. In these models the chiral field has the character of a compensating auxiliary field only rather than a collective low frequency Goldstone quarkantiquark excitation (the possibility of a nonzero quark condensate was not addressed). A general limitation of all bag and bag-like models is of course the lack of translational invariance, which is important for a realistic description of the excited states.

Common to these models is that the breaking of chiral symmetry arises from the confining interaction. This point of view contrasts with that of Manohar and Georgi [8], who pointed out that there should be two different scales in QCD, with 3 flavors. At the first one of these, $\Lambda_{\chi S B} \simeq 4 \pi f_{\pi} \simeq 1 \mathrm{GeV}$, the spontaneous breaking of the chiral symmetry occurs, and hence at distances beyond $\frac{1}{\Lambda_{\chi S B}} \simeq 0.2 \mathrm{fm}$ the valence current quarks acquire their dynamical (constituent) mass (called "chiral quarks" in [8]) and the Goldstone bosons (mesons) appear. The other scale, $\Lambda_{Q C D} \simeq 100-300 \mathrm{MeV}$, is that which characterizes confinement, and the inverse of this scale roughly coincides with the linear size of a baryon. Between these two scales then the effective Lagrangian should be formed out of the gluon fields that provide a confining mechanism as well as of the constituent quark and pseudoscalar meson fields. Manohar and Georgi did not, however, specify whether the baryons should be desrcibed as bound qqq states or as chiral solitons.

The chiral symmetry breaking scale above fits well with that which appears in the instanton liquid picture of the QCD vacuum [9, 10]. In this model the quark condensates (i.e. equilibrium of virtual quark-antiquark pairs in the vacuum state) as well as the gluon condensate are supported by instanton fluctuations of a size $\sim 0.3 \mathrm{fm}$. Diakonov and Petrov [10, 16] suggested that at low momenta (i.e. beyond the chiral symmetry breaking scale) QCD should be approximated by an effective chiral Lagrangian of the sigma-model type that contains valence quarks with dynamical (constituent) masses and meson fields. They considered a nucleon as three constituent quarks moving independently of one another in a self-consistent chiral field of the hedgehog form (mean field approximation) [15, 16]. In this picture the excited baryon states appear as rotational excitations and no explicit confining interaction is included. A very similar description for the nucleon was suggested within so called "chiral quark models" [17, 18].

The spontaneous breaking of chiral symmetry and its consequences - the dynamical quark mass generation, the appearance of the quark condensate and pseudoscalar mesons as Goldstone excitations are well illustrated by the Nambu and Jona-Lasinio model [19, 20]. This model lacks a confining interaction, which as argued below is essential for a realistic description of the properties of the baryon physics. 
derive the chiral quark model like Lagrangian [21, 22]. Here baryon was considered like above in the mean field approximation.

\section{THE CHIRAL BOSON EXCHANGE INTERACTION}

In an effective chiral symmetric description of baryon structure based on the constituent quark model the coupling of the quarks and the pseudoscalar Goldstone bosons will (in the $S U(3)_{F}$ symmetric approximation) have the form $i g \bar{\psi} \gamma_{5} \vec{\lambda}^{F} \cdot \vec{\phi} \psi$, where $\psi$ is the fermion constituent quark field operator and $\vec{\phi}$ the octet boson field operator, and $\mathrm{g}$ is a coupling constant. A coupling of this form in a nonrelativistic reduction for a constituent quark spinors will - to lowest order - give rise to a Yukawa interaction between the constituent quarks, the spin-spin component of which has the form

$$
V_{Y}\left(r_{i j}\right)=\frac{g^{2}}{4 \pi} \frac{1}{3} \frac{1}{4 m_{i} m_{j}} \vec{\sigma}_{i} \cdot \vec{\sigma}_{j} \vec{\lambda}_{i}^{F} \cdot \vec{\lambda}_{j}^{F}\left\{\mu^{2} \frac{e^{-\mu r_{i j}}}{r_{i j}}-4 \pi \delta\left(\vec{r}_{i j}\right)\right\} .
$$

Here $m_{i}$ and $m_{j}$ denote masses of the interacting quarks and $\mu$ that of the meson. There will also be an associated tensor component, which is discussed in ref. [2].

At short range the simple form (4.1) of the chiral boson exchange interaction cannot be expected to be realistic, and should only be taken to be suggestive. Because of the finite spatial extent of both the constituent quarks and the pseudoscalar mesons that the delta function in (4.1) should be replaced by a finite function, with a range of $0.6-0.7 \mathrm{fm}$ as suggested by the spatial extent of the mesons. In addition the radial behaviour of the Yukawa potential (4.1) is valid only if the boson field satisfies linear Klein-Gordon equation. The chiral symmetry requirements for the effective chiral Lagrangian (which in fact is not known), which contains constituent quarks as well as boson fields imply that these boson fields cannot be described by linear equations near their source. Therefore it is only at large distances where the amplitude of the boson fields is small that the quark-quark interaction reduces to the simple Yukawa form. At this stage the proper procedure should be to avoid further specific assumptions about the short range behavior of $V(r)$ in (1.1) and instead to extract the required matrix elements of it from the baryon spectrum and to reconstruct by this an approximate radial form of $V(r)$. The overall - sign in the effective chiral boson interaction in (1.1) corresponds to that of this short range term in the Yukawa interaction.

The flavor structure of the pseudoscalar octet exchange interaction in (1.1) between two quarks $\mathrm{i}$ and $\mathrm{j}$ should be understood as follows

$$
V\left(r_{i j}\right) \overrightarrow{\lambda_{i}^{F}} \cdot \overrightarrow{\lambda_{j}^{F}} \vec{\sigma}_{i} \cdot \vec{\sigma}_{j}=\left(\sum_{a=1}^{3} V_{\pi}\left(r_{i j}\right) \lambda_{i}^{a} \lambda_{j}^{a}+\sum_{a=4}^{7} V_{K}\left(r_{i j}\right) \lambda_{i}^{a} \lambda_{j}^{a}+V_{\eta}\left(r_{i j}\right) \lambda_{i}^{8} \lambda_{j}^{8}\right) \vec{\sigma}_{i} \cdot \vec{\sigma}_{j} .
$$

The first term in (4.2) represents the pion-exchange interaction, which acts only between light quarks. The second term represents the kaon exchange interaction, which takes place in u-s and d-s pair states. The $\eta$ - exchange, which is represented by the third term, is allowed in all quark pair states. In the $S U(3)_{F}$ symmetric limit the constituent quark masses would be equal $\left(m_{u}=m_{d}=m_{s}\right)$, the pseudoscalar octet would be degenerate and the meson-constituent quark coupling constant would be flavor independent. In this limit the form of the pseudoscalar exchange interaction reduces to (1.1), which does not break the $S U(3)_{F}$ invariance of the baryon spectrum. Beyond this limit the pion, kaon and $\eta$ exchange interactions will differ $\left(V_{\pi} \neq V_{K} \neq V_{\eta}\right)$ because of the difference between the strange and $\mathrm{u}, \mathrm{d}$ quark constituent masses $\left(m_{u, d} \neq m_{s}\right)$, and because of the mass splitting within the pseudoscalar octet $\left(\mu_{\pi} \neq \mu_{K} \neq \mu_{\eta}\right)$ (and possibly also because of flavor dependence in the meson-quark coupling constant). The source of both the $S U(3)_{F}$ symmetry breaking constituent quark mass differences and the $S U(3)_{F}$ symmetry breaking mass splitting of the pseudoscalar octet is the explicit chiral symmetry breaking in QCD. 


$$
\begin{gathered}
<\left[f_{i j}\right]_{F} \times\left[f_{i j}\right]_{S}:\left[f_{i j}\right]_{F S}\left|-V\left(r_{i j}\right) \vec{\lambda}_{i}^{F} \cdot \vec{\lambda}_{j}^{F} \vec{\sigma}_{i} \cdot \vec{\sigma}_{j}\right|\left[f_{i j}\right]_{F} \times\left[f_{i j}\right]_{S}:\left[f_{i j}\right]_{F S}> \\
=\left\{\begin{array}{rr}
-\frac{4}{3} V\left(r_{i j}\right) & {[2]_{F},[2]_{S}:[2]_{F S}} \\
-8 V\left(r_{i j}\right) & {[11]_{F},[11]_{S}:[2]_{F S}} \\
4 V\left(r_{i j}\right) & {[2]_{F},[11]_{S}:[11]_{F S}} \\
\frac{8}{3} V\left(r_{i j}\right) & {[11]_{F},[2]_{S}:[11]_{F S}}
\end{array}\right.
\end{gathered}
$$

From these the following important properties may be inferred:

(i) At short range where $V\left(r_{i j}\right)$ is positive the chiral interaction (1.1) is attractive in the symmetrical FS pairs and repulsive in the antisymmetrical ones. At large distances the potential function $V\left(r_{i j}\right)$ becomes negative and the situation is reversed.

(ii) At short range among the $F S$-symmetrical pairs the flavor antisymmetrical pairs experience a much larger attractive interaction than the flavor-symmetrical ones and among the FS-antisymmetrical pairs the strength of the repulsion in flavor-antisymmetrical pairs is considerably weaker than in symmetrical ones.

Given these properties we conclude that with the given flavor symmetry the more symmetrical $F S$ Young pattern for a baryon - the more attractive contribution at short range comes from the interaction (1.1). With two identical flavor-spin Young patterns $[f]_{F S}$ the attractive contribution at short range is larger in the case with the more antisymmetrical flavor Young pattern $[f]_{F}$.

Thus the $[3]_{F S}$ state in the $N(1440), \Delta(1600)$ and $\Sigma(1660)$ positive parity resonances from the $N=2$ band feels a much stronger attractive interaction than the mixed symmetry state $[21]_{F S}$ in the $N(1535)$, $\Delta(1700)$ and $\sum(1750)$ resonances $(N=1$ shell). Consequently the masses of the positive parity states $N(1440), \Delta(1600)$ and $\Sigma(1660)$ are shifted down relative to the other ones, which explains the reversal of the otherwise expected "normal ordering". The situation is different in the case of the $\Lambda(1405)$ and $\Lambda(1600)$, as the flavor state of the $\Lambda(1405)$ is totally antisymmetric. Because of this the $\Lambda(1405)$ gains an attractive energy, which is comparable to that of the $\Lambda(1600)$, and thus the ordering suggested by the confining oscillator interaction is maintained.

If the confining interaction in each quark pair is taken to have the harmonic oscillator form, the exact eigenvalues and eigenstates to the coinfining $3 q$ Hamiltonian are

$$
\begin{gathered}
E=(N+3) \hbar \omega+3 V_{0}, \\
\Psi=\mid N(\lambda \mu) L[f]_{X}[f]_{F S}[f]_{F}[f]_{S}>,
\end{gathered}
$$

where $N$ is the number of quanta in the state, the Elliott symbol $(\lambda \mu)$ characterizes the $S U(3)$ harmonic oscillator symmetry, and $L$ is the orbital momentum. The spatial $(X)$, flavor-spin $(F S)$, flavor $(F)$, and spin $(S)$ permutational symmetries are indicated by corresponding Young patterns (diagrams) $[f]$. All these functions are well known (see e.g. [23]). Note that the color state $[111]_{C}$, which is common to all the states, has been suppressed in (5.3). By the Pauli principle $[f]_{X}=[f]_{F S}$.

The full Hamiltonian is the sum of the confining Hamiltonian and the chiral field interaction (1.1). When the boson exchange interaction (1.1) is treated in first order perturbation theory the mass of the baryon states takes the form

$$
M=M_{0}+N \hbar \omega+\delta M_{\chi}
$$

where the chiral interaction contribution is $\delta M_{\chi}=<\Psi\left|H_{\chi}\right| \Psi>$, and $M_{0}=\sum_{i=1}^{3} m_{i}+3\left(V_{0}+\hbar \omega\right)$. The chiral interaction contribution for each baryon is a linear combination of the matrix elements of the two-body potential $V\left(r_{12}\right)$, defined as $P_{n l}=<\varphi_{n l m}\left(\vec{r}_{12}\right)\left|V\left(r_{12}\right)\right| \varphi_{n l m}\left(\vec{r}_{12}\right)>$. Here $\varphi_{n l m}\left(\vec{r}_{12}\right)$ represents the oscillator wavefunction with $\mathrm{n}$ excited quanta. As we shall only consider the baryon states in the $N \leq 2$ bands we shall only need the 4 radial matrix elements $P_{00}, P_{11}, P_{20}$ and $P_{22}$ for the numerical construction of the spectrum. 
of the matrix elements $P_{n l}$ are listed in Tables 1 and 2. In this approximate $S U(3)_{F}$-invariant version of the chiral boson exchange interaction the $\Lambda-N$ and the $\Xi-\Sigma$ mass differences would solely be ascribed the mass difference between the s and u,d quarks since all these baryons have identical orbital structure and permutational symmetries and the states in the $\Lambda$-spectrum would be degenerate with the corresponding states in the $\Sigma$-spectrum which have equal symmetries.

Table 1 The structure of the nucleon and $\Delta$ resonance states up to $N=2$, including 11 predicted unobserved or nonconfirmed states indicated by question marks. The predicted energy values (in $\mathrm{MeV}$ ) are given in the brackets under the empirical ones.

\begin{tabular}{|c|c|c|c|}
\hline$N(\lambda \mu) L[f]_{X}[f]_{F S}[f]_{F}[f]_{S}$ & LS multiplet & $\begin{array}{l}\text { average } \\
\text { energy }\end{array}$ & $\delta M_{\chi}$ \\
\hline $0(00) 0[3]_{X}[3]_{F S}[21]_{F}[21]_{S}$ & $\frac{1}{2}^{+}, N$ & 939 & $-14 P_{00}$ \\
\hline $0(00) 0[3]_{X}[3]_{F S}[3]_{F}[3]_{S}$ & $\frac{3}{2}^{+}, \Delta$ & $\begin{array}{l}1232 \\
\text { (input) }\end{array}$ & $-4 P_{00}$ \\
\hline $2(20) 0[3]_{X}[3]_{F S}[21]_{F}[21]_{S}$ & $\frac{1}{2}^{+}, N(1440)$ & $\begin{array}{l}1440 \\
\text { (input) }\end{array}$ & $-7 P_{00}-7 P_{20}$ \\
\hline $1(10) 1[21]_{X}[21]_{F S}[21]_{F}[21]_{S}$ & $\frac{1}{2}^{-}, N(1535) ; \frac{3}{2}^{-}, N(1520)$ & $\begin{array}{l}1527 \\
\text { (input) }\end{array}$ & $-7 P_{00}+5 P_{11}$ \\
\hline $2(20) 0[3]_{X}[3]_{F S}[3]_{F}[3]_{S}$ & $\frac{3}{2}^{+}, \Delta(1600)$ & $\begin{array}{l}1600 \\
\text { (input) }\end{array}$ & $-2 P_{00}-2 P_{20}$ \\
\hline $1(10) 1[21]_{X}[21]_{F S}[3]_{F}[21]_{S}$ & $\frac{1}{2}^{-}, \Delta(1620) ; \frac{3}{2}^{-}, \Delta(1700)$ & $\begin{array}{l}1660 \\
(1719)\end{array}$ & $-2 P_{00}+6 P_{11}$ \\
\hline $1(10) 1[21]_{X}[21]_{F S}[21]_{F}[3]_{S}$ & $\begin{array}{l}\frac{1}{2}^{-}, N(1650) ; \frac{3}{2}^{-}, N(1700) \\
\frac{5}{2}^{-}, N(1675)\end{array}$ & $\begin{array}{l}1675 \\
(1629)\end{array}$ & $-2 P_{00}+4 P_{11}$ \\
\hline $2(20) 2[3]_{X}[3]_{F S}[3]_{F}[3]_{S}$ & $\begin{array}{l}\frac{1}{2}^{+}, \Delta(1750 ?) ; \frac{3}{2}^{+}, \Delta(?) \\
\frac{5}{2}^{+}, \Delta(?) ; \frac{7}{2}^{+}, \Delta(?)\end{array}$ & $\begin{array}{l}1750 ? \\
(1675)\end{array}$ & $-2 P_{00}-2 P_{22}$ \\
\hline $2(20) 2[3]_{X}[3]_{F S}[21]_{F}[21]_{S}$ & $\frac{3}{2}^{+}, N(1720) ; \frac{5}{2}^{+}, N(1680)$ & $\begin{array}{l}1700 \\
\text { (input) }\end{array}$ & $-7 P_{00}-7 P_{22}$ \\
\hline $2(20) 0[21]_{X}[21]_{F S}[21]_{F}[21]_{S}$ & $\frac{1}{2}^{+}, N(1710)$ & $\begin{array}{l}1710 \\
(1778)\end{array}$ & $-\frac{7}{2} P_{00}-\frac{7}{2} P_{20}+5 P_{11}$ \\
\hline $2(20) 0[21]_{X}[21]_{F S}[21]_{F}[3]_{S}$ & $\frac{3}{2}^{+}, N(?)$ & $\begin{array}{l}? \\
(1813)\end{array}$ & $-P_{00}-P_{20}+4 P_{11}$ \\
\hline $2(20) 2[21]_{X}[21]_{F S}[21]_{F}[21]_{S}$ & $\frac{3}{2}^{+}, N(1900 ?) ; \frac{5}{2}^{+}, N(2000 ?)$ & $\begin{array}{l}1950 ? \\
(1909)\end{array}$ & $-\frac{7}{2} P_{00}-\frac{7}{2} P_{22}+5 P_{11}$ \\
\hline $2(20) 2[21]_{X}[21]_{F S}[21]_{F}[3]_{S}$ & $\begin{array}{l}\frac{1}{2}^{+}, N(?) ; \frac{3}{2}^{+}, N(?) \\
\frac{5}{2}^{+}, N(?) ; \frac{7}{2}^{+}, N(1990 ?)\end{array}$ & $\begin{array}{l}1990 ? \\
(1850)\end{array}$ & $-P_{00}-P_{22}+4 P_{11}$ \\
\hline $2(20) 0[21]_{X}[21]_{F S}[3]_{F}[21]_{S}$ & $\frac{1}{2}^{+}, \Delta(1910)$ & $\begin{array}{l}1910 \\
(1903)\end{array}$ & $-P_{00}-P_{20}+6 P_{11}$ \\
\hline $2(20) 2[21]_{X}[21]_{F S}[3]_{F}[21]_{S}$ & $\frac{3}{2}^{+}, \Delta(1920) ; \frac{5}{2}^{+}, \Delta(1905)$ & $\begin{array}{l}1912 \\
(1940)\end{array}$ & $-P_{00}-P_{22}+6 P_{11}$ \\
\hline
\end{tabular}


unobserved or nonconfirmed states indicated by question marks. The predicted energies (in $\mathrm{MeV}$ ) are given in the brackets under the empirical values.

\begin{tabular}{|c|c|c|c|}
\hline$N(\lambda \mu) L[f]_{X}[f]_{F S}[f]_{F}[f]_{S}$ & LS multiplet & $\begin{array}{l}\text { average } \\
\text { energy }\end{array}$ & $\delta M_{\chi}$ \\
\hline $0(00) 0[3]_{X}[3]_{F S}[21]_{F}[21]_{S}$ & $\frac{1}{2}^{+}, \Lambda$ & 1115 & $-14 P_{00}$ \\
\hline $1(10) 1[21]_{X}[21]_{F S}[111]_{F}[21]_{S}$ & $\frac{1}{2}^{-}, \Lambda(1405) ; \frac{3}{2}^{-}, \Lambda(1520)$ & $\begin{array}{l}1462 \\
(1512)\end{array}$ & $-12 P_{00}+4 P_{11}$ \\
\hline $2(20) 0[3]_{X}[3]_{F S}[21]_{F}[21]_{S}$ & $\frac{1}{2}^{+}, \Lambda(1600)$ & $\begin{array}{l}1600 \\
(1616)\end{array}$ & $-7 P_{00}-7 P_{20}$ \\
\hline $1(10) 1[21]_{X}[21]_{F S}[21]_{F}[21]_{S}$ & $\frac{1}{2}^{-}, \Lambda(1670) ; \frac{3}{2}^{-}, \Lambda(1690)$ & $\begin{array}{l}1680 \\
(1703)\end{array}$ & $-7 P_{00}+5 P_{11}$ \\
\hline $1(10) 1[21]_{X}[21]_{F S}[21]_{F}[3]_{S}$ & $\begin{array}{l}\frac{1}{2}^{-}, \Lambda(1800) ; \frac{3}{2}^{-}, \Lambda(?) \\
\frac{5}{2}^{-}, \Lambda(1830)\end{array}$ & $\begin{array}{l}1815 \\
(1805)\end{array}$ & $-2 P_{00}+4 P_{11}$ \\
\hline $2(20) 0[21]_{X}[21]_{F S}[111]_{F}[21]_{S}$ & $\frac{1}{2}^{+}, \Lambda(1810)$ & $\begin{array}{l}1810 \\
(1829)\end{array}$ & $-6 P_{00}-6 P_{20}+4 P_{11}$ \\
\hline $2(20) 2[3]_{X}[3]_{F S}[21]_{F}[21]_{S}$ & $\frac{3}{2}^{+}, \Lambda(1890) ; \frac{5}{2}^{+}, \Lambda(1820)$ & $\begin{array}{l}1855 \\
(1878)\end{array}$ & $-7 P_{00}-7 P_{22}$ \\
\hline $2(20) 0[21]_{X}[21]_{F S}[21]_{F}[21]_{S}$ & $\frac{1}{2}^{+}, \Lambda(?)$ & $\begin{array}{l}? \\
(1954)\end{array}$ & $-\frac{7}{2} P_{00}-\frac{7}{2} P_{20}+5 P_{11}$ \\
\hline $2(20) 0[21]_{X}[21]_{F S}[21]_{F}[3]_{S}$ & $\frac{3}{2}^{+}, \Lambda(?)$ & $\begin{array}{l}? \\
(1989)\end{array}$ & $-P_{00}-P_{20}+4 P_{11}$ \\
\hline $2(20) 2[21]_{X}[21]_{F S}[21]_{F}[3]_{S}$ & $\begin{array}{l}\frac{1}{2}^{+}, \Lambda(?) ; \frac{3}{2}^{+}, \Lambda(?) ; \\
\frac{5}{2}^{+} \Lambda(?) ; \frac{7}{2}^{+}, \Lambda(2020 ?)\end{array}$ & $\begin{array}{l}2020 ? \\
(2026)\end{array}$ & $-P_{00}-P_{22}+4 P_{11}$ \\
\hline $2(20) 2[21]_{X}[21]_{F S}[111]_{F}[21]_{S}$ & $\frac{3}{2}^{+}, \Lambda(?) ; \frac{5}{2}^{+}, \Lambda(?)$ & $\begin{array}{l}? \\
(2053)\end{array}$ & $-6 P_{00}-6 P_{22}+4 P_{11}$ \\
\hline $2(20) 2[21]_{X}[21]_{F S}[21]_{F}[21]_{S}$ & $\frac{3}{2}^{+}, \Lambda(?) ; \frac{5}{2}^{+}, \Lambda(2110)$ & $\begin{array}{l}2110 ? \\
(2085)\end{array}$ & $-\frac{7}{2} P_{00}-\frac{7}{2} P_{22}+5 P_{11}$ \\
\hline
\end{tabular}

The oscillator parameter $\hbar \omega$ and the 4 integrals are extracted from the mass differences between the nucleon and the $\Delta(1232)$, the $\Delta(1600)$ and the $N(1440)$, as well as the splittings between the nucleon and the average mass of the two pairs of states $N(1535)-N(1520)$ and $N(1720)-N(1680)$. This procedure yields the parameter values $\hbar \omega=157.4 \mathrm{MeV}, P_{00}=29.3 \mathrm{MeV}, P_{11}=45.2 \mathrm{MeV}, P_{20}=2.7 \mathrm{MeV}$ and $P_{22}=-34.7 \mathrm{MeV}$. Given these values all other excitation energies (i.e. differences between the masses of given resonances and the corresponding ground states) of the nucleon, $\Delta$ - and $\Lambda$-hyperon spectra are predicted to within $\sim 15 \%$ of the empirical values where known, and well within the uncertainty limits of those values. Note that these matrix elements provide a quantitatively satisfactory description of the $\Lambda$-spectrum even though they are extracted from the $N-\Delta$ spectrum. The splittings of the unperturbed levels due to the flavor- and spin-dependent Goldstone boson exchange interaction (1.1) is illustrated in Fig. 1.

The relative magnitudes and signs of the numerical parameter values can be readily understood. If the potential function $V(\vec{r})$ is assumed to have the form of a Yukawa function with a smeared $\delta$-function term that is positive at short range $r \leq 0.6-0.7 \mathrm{fm}$, as suggested by the pion size $\sqrt{<r_{\pi}^{2}>}=0.66$ fm, one expects $P_{20}$ to be considerably smaller than $P_{00}$ and $P_{11}$, as the radial wavefunction for the excited S-state has a node, and as it extends further into region of where the potential is negative. The negative value for $P_{22}$ is also natural as the corresponding wavefunction is suppressed at short range and extends well beyond the expected 0 in the potential function. The relatively small value of the oscillator parameter $(157.4 \mathrm{MeV})$ leads to the empirical value $0.86 \mathrm{fm}$ for the nucleon radius $\sqrt{<r^{2}>}=\sqrt{\hbar / m \omega}$ if the light quark constituent mass is taken to be 330-340 MeV, as suggested by the magnetic moments of the nucleon. 
The model described above has relied on an interaction potential function $V(r)$ in $(1.1)$ that is flavor independent. A refined version takes into account the explicit flavor dependence of the potential function in (4.2) $\left(V_{\pi} \neq V_{K} \neq V_{\eta}\right)$. In the following we show how this explicit flavor dependence provides us with an explanation of the mass spliting between the $\Lambda$ and the $\Sigma$ which have the same quark content and the same $F S, F$ and $S$ symmetries, i.e. they are degenerate within the $S U(3)_{F}$ version $(1.1)$ of the chiral boson exchange interaction.

Beyond the $S U(3)_{F}$ limit the ground state baryons will be determined by the $\pi$-exchange radial integral $P_{00}^{\pi}$, the $K$-exchange one, $P_{00}^{K}$, and by the $\eta$-exchange integrals, $P_{00}^{u u}=P_{00}^{u d}=P_{00}^{d d}, P_{00}^{u s}$ and $P_{00}^{s s}$, where the superscripts indicate quark pairs to which the $\eta$-exchange applies. As indicated by the Yukawa interaction (4.1) these matrix elements should be inversely proportional to the product of the quark masses of the pair state. Thus $P_{n l}^{u s}=\frac{m_{u}}{m_{s}} P_{n l}^{u u}, \quad P_{n l}^{s s}=\left(\frac{m_{u}}{m_{s}}\right)^{2} P_{n l}^{u u}$. We also assume that $P_{00}^{u s} \simeq P_{00}^{K}$, which is suggested by the fact that the quark masses are equal in the states, in which these interactions act, and by the near equality of the kaon and $\eta$ masses, $\mu_{\eta} \simeq \mu_{K}$. Thus we have only two independent radial integrals.

To determine the integrals $P_{00}^{\pi}, P_{00}^{K}$ and the quark mass difference $\Delta_{q}=m_{s}-m_{u}$ we consider the $\Sigma(1385)-\Sigma, \Delta-N$ and $\Lambda-N$ splittings:

$$
\begin{gathered}
m_{\Sigma(1385)}-m_{\Sigma}=4 P_{00}^{u s}+6 P_{00}^{K}, \\
m_{\Delta}-m_{N}=12 P_{00}^{\pi}-2 P_{00}^{u u}, \\
m_{\Lambda}-m_{N}=6 P_{00}^{\pi}-6 P_{00}^{K}+\Delta_{q},
\end{gathered}
$$

which imply $P_{00}^{K}=19.6 \mathrm{MeV}, \Delta_{q}=121 \mathrm{MeV}$ if the conventional value $340 \mathrm{MeV}$ is given to $m_{u}$, $P_{00}^{\pi}=28.9 \mathrm{MeV}$ and the quark mass ratio $m_{s} / m_{u}=1.36$. These matrix element values lead to the values $65 \mathrm{MeV}$ and $139 \mathrm{MeV}$ for the $\Sigma-\Lambda$ and the $\Xi-\Sigma$ mass differences

$$
\begin{gathered}
m_{\Sigma}-m_{\Lambda}=8 P_{00}^{\pi}-4 P_{00}^{K}-\frac{4}{3} P_{00}^{u u}-\frac{8}{3} P_{00}^{u s}, \\
m_{\Xi}-m_{\Sigma}=P_{00}^{\pi}+\frac{1}{3} P_{00}^{u u}-\frac{4}{3} P_{00}^{s s}+\Delta_{q}
\end{gathered}
$$

in good agreement with the empirical values $77 \mathrm{MeV}$ and $125 \mathrm{MeV}$ respectively.

\section{EXCHANGE CURRENT CORRECTIONS TO THE MAGNETIC MOMENTS}

A flavor dependent interaction of the form (1.1) will imply the presence of an irreducible two-body exchange current operator, as seen e.g. directly from the continuity equation, by which the commutator of the interaction and the single particle charge operator equals the divergence of the exchange current density [24]. Because this commutator vanishes with interparticle separation this exchange current is however a priori expected to be of less importance for baryons, than for nuclei, in which the longer range of the wave functions can lead to large matrix elements of the pion exchange current operator. This is one contributing reason for why the naive constituent quark model provides such a successful description of the magnetic moments.

The general form of the octet vector exchange current operator that is associated with the complete octet mediated interaction (4.2) will have the form [2]

$$
\vec{\mu}^{e x}=\mu_{N}\left\{\tilde{V}_{\pi}\left(r_{i j}\right)\left(\lambda_{i}^{1} \lambda_{j}^{2}-\lambda_{i}^{2} \lambda_{j}^{1}\right)+\tilde{V}_{K}\left(r_{i j}\right)\left(\lambda_{i}^{4} \lambda_{j}^{5}-\lambda_{i}^{5} \lambda_{j}^{4}\right)\right\}\left(\vec{\sigma}_{i} \times \vec{\sigma}_{j}\right) .
$$

Here $\tilde{V}_{\pi}(r)$ and $\tilde{V}_{K}(r)$ are dimensionless functions that describe $\pi$ and $K$ exchange respectively and which includes at long range both the pionic (kaonic) current and the pair current term. 
and their experimental values are listed in Table 3 (columns "IA" and "exp", respectively). A natural approach is to determine the mass ratios $m_{N} / m_{u}$ and $m_{N} / m_{s}$ to fit the experimental values of the magnetic moments of the $\Sigma^{-}$and $\Xi^{-}$octet and the $\Omega$ and $\Delta^{++}\left(\mu_{\Omega}=-2.019 \pm 0.054 \mu_{N}, \mu_{\Delta^{++}}=\right.$ $4.52 \pm 0.50 \mu_{N}$ ) decuplet baryons, which are unaffected by the exchange current operator (7.1). While with only two independent variables it is not possible to fit all four experimental magnetic moments exactly, the best overall fit $\mu_{\Sigma^{-}}=-1.00 \mu_{N}, \mu_{\Xi^{-}}=-0.59 \mu_{N}, \mu_{\Omega^{-}}=-2.01 \mu_{N}, \quad \mu_{\Delta^{++}}=5.52 \mu_{N}$ happens to be obtained with precisely the ratios $m_{N} / m_{u}=2.76$ and $m_{N} / m_{s}=2.01$, which used for constituent quark masses to fit ground state baryons $\left(m_{u}=340 \mathrm{MeV}\right.$ and $\left.m_{s}=467 \mathrm{MeV}\right)$.

We find (see Table 3) that the meson exchange current contributions systematically improve predictions of the naive constituent quark model (i.e. with one-body quark currents only) for all known magnetic moments. However these contributions are not large and do not exceed $10 \%$ in agreement with the expectation above.

Table 3 Magnetic moments of the baryon octet (in nuclear magnetons). Column IA contains the quark model impulse approximation expressions, column "exp" the experimental values, column I the impulse approximation predictions, column II the exchange current contribution with $<\varphi_{000}\left(\vec{r}_{12}\right)\left|\tilde{V}_{\pi}\left(r_{12}\right)\right| \varphi_{000}\left(\vec{r}_{12}\right)>=-0.018$ and $<\varphi_{000}\left(\vec{r}_{12}\right)\left|\tilde{V}_{K}\left(r_{12}\right)\right| \varphi_{000}\left(\vec{r}_{12}\right)>=0.03$, and column III the net predictions. All magnetic moments are given in nuclear magnetons.

\begin{tabular}{|l|l|l|l|l|l|}
\hline & IA & exp & I & II & III \\
\hline$p$ & $\frac{m_{N}}{m_{u}}$ & +2.79 & +2.76 & +0.07 & +2.83 \\
$n$ & $-\frac{2}{3} \frac{m_{N}}{m_{u}}$ & -1.91 & -1.84 & -0.07 & -1.91 \\
$\Lambda$ & $-\frac{1}{3} \frac{m_{N}}{m_{s}}$ & -0.61 & -0.67 & +0.06 & -0.61 \\
$\Sigma^{+}$ & $\frac{8}{9} \frac{m_{N}}{m_{u}}+\frac{1}{9} \frac{m_{N}}{m_{s}}$ & +2.42 & +2.68 & -0.12 & +2.56 \\
$\Sigma^{0}$ & $\frac{2}{9} \frac{m_{N}}{m_{u}}+\frac{1}{9} \frac{m_{N}}{m_{s}}$ & $?$ & +0.84 & -0.06 & +0.72 \\
$\Sigma^{0} \rightarrow \Lambda$ & $-\frac{1}{\sqrt{3}} \frac{m_{N}}{m_{u}}$ & $|1.61|$ & -1.59 & -0.01 & -1.60 \\
$\Sigma^{-}$ & $-\frac{4}{9} \frac{m_{N}}{m_{u}}+\frac{1}{9} \frac{m_{N}}{m_{s}}$ & -1.16 & -1.00 & 0 & -1.00 \\
$\Xi^{0}$ & $-\frac{2}{9} \frac{m_{N}}{m_{u}}-\frac{4}{9} \frac{m_{N}}{m_{s}}$ & -1.25 & -1.51 & +0.12 & -1.39 \\
$\Xi^{-}$ & $\frac{1}{9} \frac{m_{N}}{m_{u}}-\frac{4}{9} \frac{m_{N}}{m_{s}}$ & -0.65 & -0.59 & 0 & -0.59 \\
\hline
\end{tabular}

The pion exchange current contribution to the magnetic moments is also discussed within the hybrid model (with the strong gluon exchange contribution) [25] which, however, fails to explain excited baryon masses.

\section{RESOLUTION OF THE $\mathbf{N}^{*} \rightarrow \mathbf{N} \eta$ PUZZLE}

We shall suggest here a simple explanation for a strong selectivity of the $N \eta(\Lambda \eta$ and $\Sigma \eta)$ decay branching ratios of the $N^{*}\left(\Lambda^{*}\right.$ and $\left.\Sigma^{*}\right)$ baryons and at the same time an absence of such a selectivity for the $N \pi(N K)$ decays within the chiral quark model outlined above [3]. 
quarks in the $S_{i j}=T_{i j}=0$ and strong repulsion in the $S_{i j}=1, T_{i j}=0$ and $S_{i j}=0, T_{i j}=1$ channels. The chiral field interaction is attractive, but rather weak, in the $S_{i j}=T_{i j}=1$ pair state. Thus those baryons which contain the $S_{i j}=T_{i j}=0$ quark pair state will be strongly clusterized into quark-diquark configuration with $S_{12}=T_{12}=0$ diquark quantum numbers. These will be all baryons with $[21]_{F}[21]_{S}$ symmetries of zero order wave function and with the smallest possible orbital momentum $L=0$ or 1 ( $N$, $N(1440), N(1535)$ and $N(1710))$. The baryons with the $[21]_{F}[3]_{S}$ symmetries $(\mathrm{N}(1650), \ldots)$ have the quark pair components $S_{i j}=T_{i j}=1$ and $S_{i j}=1, T_{i j}=0$. In the former there is rather soft attraction and in the latter there is much larger repulsive interaction at short range. This will again lead to a clusterization into quark-diquark configuration but with $S_{i j}=T_{i j}=1$ quantum numbers for diquark. It is a clusterization into quark-diquark configurations with different quantum numbers of diquarks in different baryons which provides the sought explanation of the $\eta$ decay puzzle.

Consider first the case of the $N(1650)$. As in $\eta$ decay the isospin of the involved quark is unchanged it cannot proceed through the $S_{12}=1, T_{12}=1 \rightarrow S_{12}=0, T_{12}=0$ transition that would connect the diquark states in the $N(1650)$ and the nucleon. The corresponding pion decay, in which isospin flip is possible, can on the other hand connect these pair states. The reason for the large $\eta$ decay branch of the $N(1535)$ in contrast is that its wavefunction has diquark with the same spin-isospin structure as the nucleon.

This argument generalizes to the predictions that: (i) all baryon resonances above the corresponding $\eta$ decay threshold with $[21]_{F S}[21]_{F}[21]_{S}$ symmetry zero order wavefunctions and smallest possible orbital and total angular momentum $\left(\frac{1}{2}^{-}, N(1535) ; \frac{1}{2}^{+}, N(1710) ; \frac{1}{2}^{-}, \Lambda(1670) ; \frac{1}{2}^{-}, \Sigma(1750)\right)$ should have large $\eta$-decay branching ratios whereas (ii) the baryon resonances that have $[21]_{F S}[21]_{F}[3]_{S}$ symmetry zero order wave functions should have strongly suppressed $\eta$-decay branching ratios. This prediction is in excellent agreement with the corresponding empirical branching ratios, all of which are large for the baryons in the list (i) and vanish for all other baryons.

\section{CONCLUSION}

It proves instructive to consider the symmetry structure of the harmonic confining + chiral octet mediated interaction (1.1) model presented here in view of the highly satisfactory predictions obtained for the baryon spectra. The symmetry group for the orbital part of a harmonically bound system of 3 quarks is $U(6)$. In the absence of the fine-structure interaction (1.1), and with equal $\mathrm{u}, \mathrm{d}$ and s- quark masses, the baryon states would form unsplit multiplets of the full symmetry group $S U(6)_{F S} \times U(6)_{\text {conf }}$. The $S U(3)_{F}$ symmetrical version of the chiral interaction (1.1) reduces this degeneracy within the multiplets to those that corresponding to $S U(3)_{F} \times S U(2)_{S} \times U(6)_{c o n f}$ and is in fact strong enough to shift some of the $\mathrm{N}=2$ states below the $\mathrm{N}=1$ states and to mix positions of different multiplets. Thus the $\mathrm{N}=2$ resonance $N(1440)$ is shifted down below the $\mathrm{N}=1$ resonance $N(1535)$ etc. When this shifting moves states from adjacent N-levels close to each other near degenerate parity doublets appear. The model thus suggests an explicit explanation of the observed near parity doubling of the spectrum already in the Nambu-Goldstone mode.

The mass splittings between the different members of the same $S U(3)_{F} \times S U(2)_{S} \times U(6)_{\text {conf }}$ multiplet arise due to both the constituent quark mass difference and the different strength of the meson-exchange interaction $V_{\pi} \neq V_{K} \neq V_{\eta}$ beyond the $S U(3)_{F}$ limit. Thus even those states in the $\Lambda$ and $\Sigma$ spectrum which have identical quark content and equal spatial, flavor, spin and flavor-spin symmetries, get different contributions from the interaction (4.2) and consequently different masses.

There is no fundamental reason for why the effective confining interaction between the constituent quarks should have to be harmonic. The low lying part of the baryon spectrum depends to a higher degree on the chiral boson exchange interaction than on the confining interaction. This can be illustrated by the fact that only about a quarter of the mass difference between the nucleon and the lowest $\frac{1}{2}^{-}$ state $N(1535)$ is due to the confining central interaction, whereas the remaining 3 quarters are due to the spin-spin interaction (1.1). However the ground state oscilations contribution $3 \hbar \omega$ to the nucleon 
$m^{\prime}=m+\hbar \omega$, as in this case (i.e. with the large constituent quark mass) one could not describe baryon magnetic moments. The low-lying part of the baryon spectrum is not very sensitive to the form of the confining interaction, but the very satisfactory numerical predictions obtained here for the baryon spectra up to about $1 \mathrm{GeV}$ excitation energy suggest that any anharmonic corrections should be small. Quantitative study of the detailed form of the confining interaction would require a simultaneous specification of the detailed short range part of the chiral interaction (1.1), and would presumably also need increased accuracy for the empirical resonance energies. If the harmonic confining interaction is replaced by a nonharmonic form, the $U(6)$ spatial symmetry of the confining form is reduced to $O(3)$.

Be it as it may, the present organization of fine structure of the baryon spectrum based on the quarkquark interaction that is mediated by the octet of pseudoscalar mesons, which represent the Goldstone bosons associated with the hidden mode of the approximate chiral symmetry of QCD is both simple and phenomenologically successful. The predicted energies of the states in the nucleon and strange hyperon spectra agree with the empirical values, where known, to within a few percent. This interaction between light and strange quarks inside the charm and bottom hyperons with one heavy quark is also of crucial importance for those baryons [4].

The very satisfactory predictions obtained here for the baryon spectrum reconcile the quark model for baryons with the phenomenologically successful meson exchange description of the nucleon-nucleon interaction.

Finally, mention should be made that the coupling between the constituent quarks and the pseudoscalar mesons governs the structure and content of the quark sea. It has recently been shown to resolve well known problems that are associated with the strangeness content of the nucleon and the nucleon spin structure as measured in deep inelastic lepton-proton scattering [26, 27].

\section{References}

[1] L. Ya. Glozman and D. O. Riska, The Baryon Spectrum and Chiral Dynamics, Preprint HU-TFT-94-47, [LANL hep-ph 9411279] (1994); Systematics of the Light and Strange Baryons and the Symmetries of QCD, Preprint HU-TFT-94-48, [LANL hep-ph 9412231] (1994).

[2] L. Ya. Glozman and D. O. Riska, The Spectrum of the Nucleons and the Strange Hyperons and Chiral Dynamics, Preprint DOE/ER/40561-187-INT95-16-01 [LANL hep-ph 9505422], Physics Reports (1995) in print.

[3] L. Ya. Glozman and D. O. Riska, Quark Model Explanation of the $N^{*} \rightarrow N \eta$ Branching Ratios, Preprint HU-TFT-95-49, [LANL hep-ph 9508327], Physics Letters - in print.

[4] L. Ya. Glozman and D. O. Riska, The Charm and Bottom Hyperons and Chiral Dynamics, Preprint HU-TFT-95-53, [LANL hep-ph 9509269] (1995).

[5] S. Weinberg, Phys. Rev. D11 (1975) 3583.

[6] G.'t Hooft, Phys. Rev. D14 (1976) 3432.

[7] S. Weinberg, Physica 96 A (1979) 327.

[8] A. Manohar and H. Georgi, Nucl. Phys. B234, (1984) 189.

[9] E.V. Shuryak, Phys.Rep. C115 (1984) 152.

[10] D. I. Diakonov, V. Yu. Petrov, Phys. Lett. B147 (1984) 351; Nucl. Phys. B272 (1986) 457.

[11] A. DeRujula, H. Georgi and S. L. Glashow, Phys. Rev. D12 (1975) 147.

[12] N. Isgur and G. Karl, Phys. Rev. D18 (1978) 4187; D19 (1979) 2653. B147 (1984) 351; Nucl. Phys. B272 (1986) 457. 
A. W. Thomas, J. Phys. G7 (1981) L283.

[14] W. Weise, International Review of Nucl. Phys., 1 (1984) 116; D. Robson, Topical Conference on Nuclear Chromodynamics, J. Qiu and D. Sivers (eds.), World Scientific, Singapore, 174 (1988).

[15] D. I. Diakonov, V. Yu. Petrov and P. V. Pobylitsa, Nucl. Phys. B306 (1988) 809.

[16] D. I. Diakonov, lecture at this school.

[17] S. Kahana, V. Soni and G. Ripka, Nucl. Phys. A415 (1984) 351.

[18] M. Birse and M. Banerjee, Phys. Rev. D31 (1985) 118.

[19] Y. Nambu, G. Jona-Lasinio, Phys. Rev. 122 (1961) 345.

[20] U. Vogl, W. Weise, Progr. Part. Nucl. Phys., 27 (1991) 195.

[21] H. Reinhard, lecture at this school and references therein.

[22] K. Goeke, lecture at this school and references therein.

[23] L. Ya. Glozman and E. I. Kuchina, Phys. Rev. C49 (1994) 1149.

[24] D. O. Riska, Phys. Reports 181 (1989) 207.

[25] G. Wagner, A. Buchmann, A. Faessler, contribution at this school.

[26] E.J. Eichten, I. Hinchliffe, C. Quigg, Phys. Rev. D45 (1992) 2269.

[27] T.P. Cheng, L.-F. Li, Phys. Rev. Lett. 74 (1995) 2872; hep-ph 9510235. 
This figure "fig1-1.png" is available in "png" format from: http://arxiv.org/ps/hep-ph/9511418v1 